\title{
Análise Estatística em Geofísica: Aplicação em Dados de Perfis de Poços
} Euler B. S. Marinho, IGEO/UFBA; Amin Bassrei ${ }^{1}$, CPGG/IGEO/UFBA \& INCT-GP; Roberto F. S. Andrade, IF/UFBA

Copyright 2016, SBGf - Sociedade Brasileira de Geofísica

Este texto foi preparado para a apresentação no VII Simpósio Brasileiro de Geofísica, Ouro Preto, 25 a 27 de outubro de 2016. Seu conteúdo foi revisado pelo Comitê Técnico do VII SimBGf, mas não necessariamente representa a opinião da SBGf ou de seus associados. É proibida a reprodução total ou parcial deste material para propósitos comerciais sem prévia autorização da SBGf.

\begin{abstract}
One common approach for analysis of spatial series, as in the case of well logs, is the scale property of the existing fluctuations in those series. This type of analysis allows us to identify correlations present in a single signal (autocorrelation) or in two separate signals (cross-correlation), whose interpretation depends on the signal to be studied. The interpretation of well logs is very important because it can lead to the understanding of periodic and well as nonperiodic phenomena. In this study we compare spectral analysis methods with detrended cross correlation analysis, that are used to identify and characterize correlated data obtained in spatial series by means of the cross-correlation exponents (global estimate) and coefficients (local estimate). The comparison was performed on well log data from Jequitinhonha Basin, state of Bahia, Brazil. There were some deviations in value of the spectral exponent $b$ when comparing the two methods but in general the results were consistent.
\end{abstract}

\section{Introdução}

O conhecimento da subsuperfície tem uma importância fundamental, em especial para as atividades de exploração. A constituição da subsuperfície é expressa em camadas, sendo tais camadas formadas e modificadas por processos naturais ao longo do tempo geológico (Lowrie, 1997; Dimri, 2005; Goltz, 1998).

Para entender esse sistema é necessário desvendar a sua história, ou seja, os processos que atuaram, em que condições e em quais tipos de materiais, por meio das marcas existentes em suas estruturas.

Avanços na compreensão da litosfera são obtidos através da análise de dados geológicos e geofísicos, que auxiliam na exploração de seus recursos diminuindo os riscos e tornando-a mais eficiente.

No caso do petróleo, dados de perfis de poços são particularmente de grande relevância, pois consistem no registro de propriedades físicas dos materiais da subsuperfície em função da profundidade. Dentre essas propriedades incluem-se a resistividade, atividade radioativa, temperatura, etc. Tais dados fornecem informações sobre a sequência de rochas dos poços perfurados, interfaces entre camadas e estruturas geológicas, sendo utilizados para caracterizar reservatórios de hidrocarbonetos e avaliar a viabilidade de exploração (Lowrie, 1997).

\section{DCCA e o Cálculo de Expoentes}

Sejam dois conjuntos de séries de dados $y_{k}$ e $y_{k}^{\prime}$ de mesmo tamanho $N$ cada uma, com incrementos $x_{i}$ e $x_{i}^{\prime}$ respectivamente, dados por,

$$
y_{k}=\sum_{i=1}^{k} x_{i} \text { e } y_{k}^{\prime}=\sum_{i=1}^{k} x_{i}^{\prime}
$$

onde $k=1,2,3, \ldots, N$. Cada série integrada é dividida em $N-v+1$ caixas de tamanho $v$. Em cada caixa classificado por $(m, v)$, com $1 \leq m \leq N-v+1$, é calculada a medida da flutuação que depende do método a ser utilizado.

A Figura 1 ilustra o procedimento do DCCA entre duas séries de mesmo tamanho $N$.

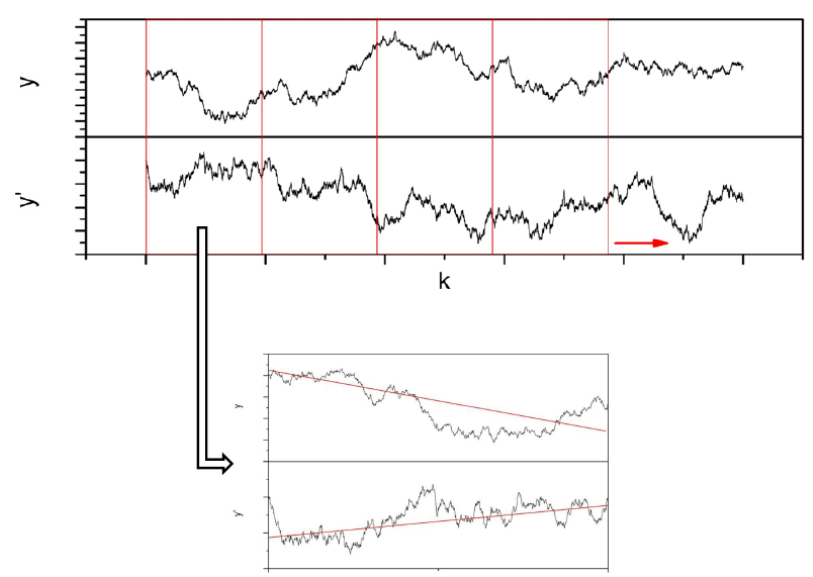

Figura 1: llustração do método DCCA. Duas séries, y e $y^{\prime}$ de mesmo tamanho $N$, estão superpostas possuindo $k$ caixas de largura $\vee$ cada uma. Na parte inferior da figura tem-se uma ampliação de uma dessas caixas mostrando o procedimento do método DCCA.

Neste trabalho, foram avaliadas quatro tipos de medidas de flutuação, respectivamente classificados como, DFA Detrended Fluctuation Analysis, equação (2), SCCA Standard Cross Correlation Analysis, equação (3), DCCA Detrended Cross Correlation Analysis, equação (4), e $|D C C A|$, equação (5):

\footnotetext{
${ }^{1}$ Atualmente como professor visitante na Universidade de Stanford.
} 


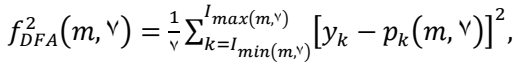

$$
\begin{aligned}
& f_{S C C A}^{2}(m, \vee)=\frac{1}{\vee} \sum_{k=I}^{I_{\max } \max (m, v)}\left[y_{k}-\bar{y}_{k}(m, \vee)\right]\left[y_{k}^{\prime}-\bar{y}_{k}^{\prime}(m, \vee)\right] \text {, } \\
& f_{D C C A}^{2}(m, \vee)=\frac{1}{\vee} \sum_{k=I_{\min (m, v)}}^{I_{\max (m, v)}}\left[y_{k}-p_{k}(m, \vee)\right]\left[y_{k}^{\prime}-p_{k}^{\prime}(m, \vee)\right] \text {, }
\end{aligned}
$$

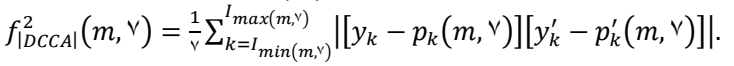

A indicação $|D C C A|$ na equação (5) usa o valor absoluto das flutuações locais de cada série. Nas equações (2) a (5), $\bar{y}$ e $\bar{y}^{\prime}$ são os valores médios de $y_{k}$ e $y_{k}^{\prime}$ na caixa $(m, \vee)$ limitada por $I_{\min (m, \vee)}$ e $I_{\max (m, \vee)} ; p_{k}(m, \vee)=$ $a(m, \vee) x_{k}+b(m, \vee)$ e $p_{k}^{\prime}\left(m,{ }^{\vee}\right)=a^{\prime}(m, \vee) x_{k}^{\prime}+b^{\prime}(m, \vee)$ são os polinômios de primeiro grau avaliados pelo método dos mínimos quadrados que expressam as tendências locais lineares da série na caixa $(m, v)$. Notese que $f_{D F A}^{2}(m, v)$ é um caso particular de $f_{D C C A}^{2}(m, v)$ ou $f_{|D C C A|}^{2}(m, \vee)$ com duas séries iguais.

A função de flutuação é calculada por cada largura $\vee$ conforme

$$
F_{X}^{2}\left({ }^{\vee}\right)=\frac{1}{N-\vee+1} \sum_{m=1}^{N-\gamma+1} f_{X}^{2}(m, \vee)
$$

onde o subíndice $X$ está relacionado ao método a ser utilizado, ou seja, $X=$ DFA, SCCA, DCCA ou |DCCA|.

Se a série apresenta propriedades de escala relacionadas às correlações cruzadas, espera-se uma lei de potência da forma $F_{X}(v) \sim v^{\lambda}$. O expoente $\lambda$ representa a medida da correlação cruzada entre duas séries analisadas. No caso do DFA, o expoente $\lambda$ torna-se equivalente ao expoente de Hurst (1951) ou rugosidade, geralmente indicado por $\mathrm{H}$ ou $\alpha$.

Os valores de $\lambda$ indicam o tipo de correlação entre as séries. Assim, como o expoente H (Feder, 1988), $\lambda$ está relacionado com 0 conceito de persistência e antipersistência. Para $\lambda>0,5$ a série é persistente, isto é, um grande (pequeno) aumento em uma das séries é seguido, em média, por um grande (pequeno) aumento no futuro das outras séries. A série será antipersistente quando $\lambda<0,5$, o que representa uma situação oposta, com ambas as séries revertendo a direção dos movimentos recentes. Finalmente, quando $\lambda=0,5$ os incrementos são, em média, não correlacionados com o anterior.

\section{DCCA e o Cálculo de Coeficientes}

O coeficiente DCCA de correlação cruzada $\sigma_{D C C A}$ foi proposto para quantificar a correlação cruzada entre duas séries não estacionárias (Podobnik et al., 2011; Zebende et al., 2012). Este coeficiente é definido para cada análise de escala $\vee$ (largura das caixas) através da razão

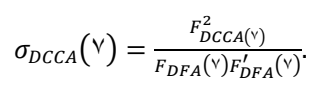

O coeficiente DCCA é uma quantidade adimensional que varia na faixa $-1 \leq \sigma_{D C C A} \leq 1$. Similar ao coeficiente de correlação padrão, $\sigma_{D C C A}=1$ indica uma perfeita correlação cruzada enquanto que $\sigma_{D C C A}=-1$ mostra uma perfeita anticorrelação. Se $\sigma_{D C C A}=0$, não há correlação cruzada entre as séries. Como o coeficiente DCCA é uma função de escala $\vee$, é possível observar como a correlação cruzada entre as séries se comporta para diferentes escalas e determinar, por exemplo, se uma alta correlação cruzada vale para todas as escalas ou se há mudança de intensidade na correlação cruzada em uma determinada escala.

\section{Dados Utilizados}

Este trabalho foi baseado em dados de perfis de poços coletados pela PETROBRAS na Bacia do Jequitinhonha, que está situada na região sudeste do estado da Bahia, e compreende uma área total de aproximadamente 4000 $\mathrm{km}^{2}$, abrangendo parte dos municípios de Belmonte, Canavieiras e Una. Os poços foram perfurados na região marinha da Bacia.

Foram utilizados três tipos de perfis coletados em quatro diferentes poços: 1BAS68, 1BAS121, 1BAS80 e 1BAS37. Estes poços podem ser visualizados no mapa da Figura 2. Os perfis analisados para cada poço são: sônico $D T\left(\frac{\mu s}{f t}\right)$, raios gama $\gamma(G A P I)$ e resistividade $\rho(\Omega m)$.

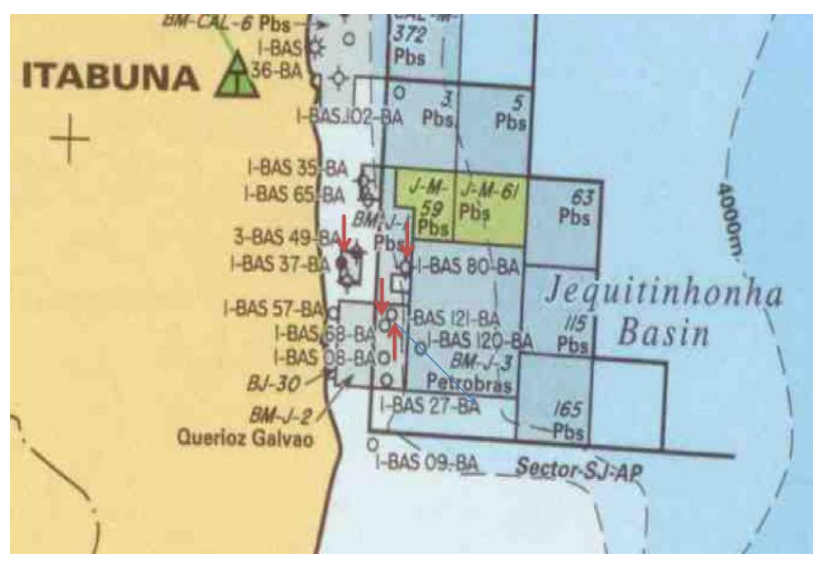

Figura 2: Mapa com a região onde os poços foram perfurados. Os poços estão identificados por setas vermelhas. Fonte: PETROBRAS.

As medições dos perfis foram feitas em intervalos de $15,24 \mathrm{~cm}$, que corresponde a distância entre dois pontos consecutivos. A profundidade máxima atingida por cada poço, em metros, está ligada a quantidade de pontos obtidos durante o processo de perfilagem, o que leva aos intervalos espaciais onde as análises podem ser efetuadas (Tabela 1 ). 


\begin{tabular}{|l|l|l|l|l|}
\hline Perfil & $\begin{array}{l}\text { Poço } \\
\text { 1BAS68 }\end{array}$ & $\begin{array}{l}\text { Poço } \\
\text { 1BAS121 }\end{array}$ & $\begin{array}{l}\text { Poço } \\
\text { 1BAS37 }\end{array}$ & $\begin{array}{l}\text { Poço } \\
\text { 1BAS80 }\end{array}$ \\
\hline$\gamma(G A P I)$ & $108-4525$ & $0-3495$ & $0-1625$ & $75-2532$ \\
\hline$D T\left(\frac{\mu s}{f t}\right)$ & $437-4525$ & $98-3495$ & $400-1625$ & $406-2532$ \\
\hline$\gamma(G A P I)$ & $446-3750$ & $90-2700$ & $400-1625$ & $406-2532$ \\
\hline
\end{tabular}

Tabela 1: Faixas de profundidades, em metros, por perfil em cada poço.

Os poços 1BAS68 e 1BAS121 estão distantes em cerca de $2,5 \mathrm{~km}$. Note que na Tabela 1 nem todas as propriedades físicas são registradas com a mesma profundidade, ou seja, o início e o final de cada registro para cada propriedade não tem necessariamente o mesmo ponto. Então, dependendo do tipo de correlação, foi realizado um ajuste no tamanho das séries, de modo a deixar com o mesmo tamanho.

A escolha dessas três propriedades petrofísicas baseiase no fato da sua relevância, pois são medidas que estão ligadas com outras propriedades físicas das rochas. A Figura 3 mostra os três perfis para o poço 1BAS121.

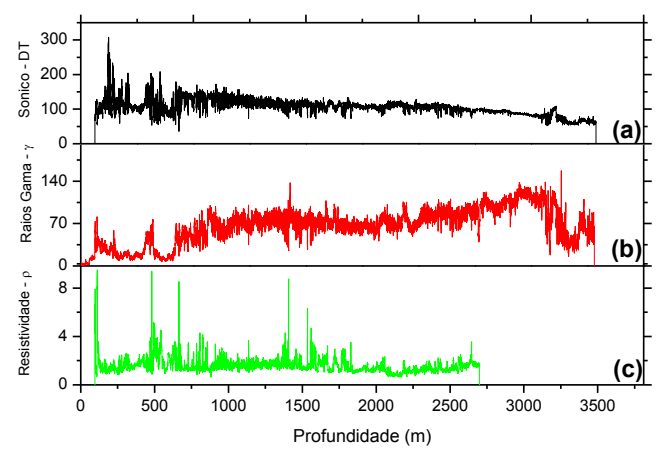

Figura 3: Registros dos perfis: (a) $D T\left(\frac{\mu s}{f t}\right)$, (b) $\gamma(G A P I) \mathrm{e}$ (c) $\rho(\Omega m)$ no poço 1BAS121 em função da profundidade (m).

\section{Análise DCCA Global}

Os conjuntos originais dos dados de perfis foram interpretados conforme as séries integradas $y_{k}$. A subtração de dois valores subsequentes conduz para séries de passos $x_{i}$, a partir da qual duas séries adicionais de passos de magnitude e de sinal podem ser obtidas (a partir da original), respectivamente definidas por $M=\left|x_{i}\right|$ e $S=\operatorname{sign}\left(x_{i}\right)$, onde as séries de sinais, dependendo do sinal de cada incremento da série original, terão valores $1,-1$ ou 0 , (Peng et al., 2001). A Figura 4 faz uma comparação entre as séries de magnitude, sinais e originais.

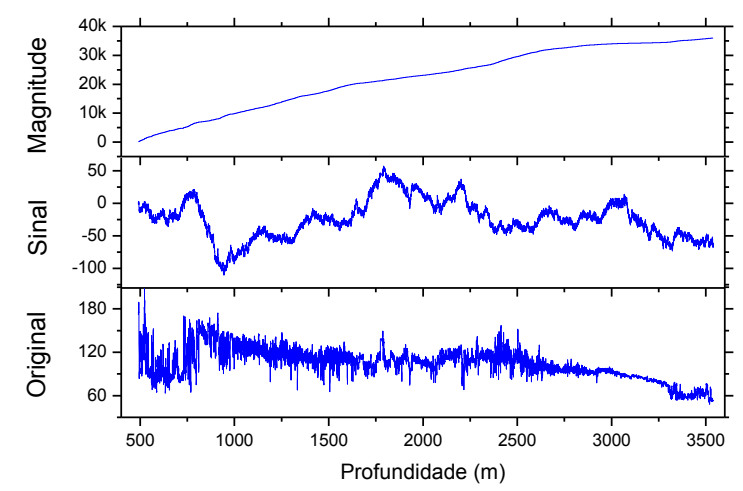

Figura 4: Comparação entre as séries de magnitude, de sinal e original do perfil sônico.

Em seguida, as séries integradas correspondentes $y_{k}^{M} \mathrm{e}$ $y_{k}^{S}$ foram calculadas por meio da equação (1). O propósito de usar a função flutuação $F_{|D C C A|}(\mathrm{V})$ no lugar de $F_{D C C A}(\mathrm{~V})$ está relacionado ao fato de que há uma redução na função flutuação causada pelas contribuições negativas devido aos possíveis diferentes sinais nos termos da equação (4). Não havendo esta troca provavelmente obteríamos gráficos sem comportamento de escala, como visto em Zebende (2011). Além disso, existe uma relação aproximada entre os expoentes, que já foi notada para conjuntos de dados distintos analisados em: $\lambda \approx \frac{H+H^{\prime}}{2}$, onde $H$ e $H^{\prime}$ são os expoentes de Hurst de cada série.

Para estes dados de perfis de poços, foram feitas análises para diferentes tamanhos das séries, ou seja, o número total de observações $N$ é dividido em sequências $\checkmark$ de diversos tamanhos. Também, adotou-se diferentes posições de partida na série para que fosse possível analisar subséries. A Figura 5 mostra a dependência de $F(\vee)$ em função de $\vee$ para as três séries: (a) Original, (b) Magnitude e (c) Sinal, que foram obtidos a partir da propriedade física raios gama. Gráficos semelhantes à Figura 5 foram obtidos para todos os parâmetros físicos para os quatro poços estudados.

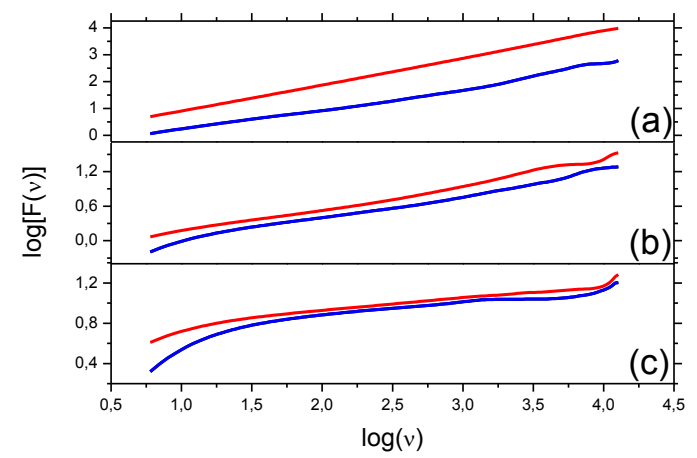

Figura 5: Análise DCCA, para a autocorrelação, no poço 1BAS121 para o perfil gama: $F_{D F A}$ (preto), $F_{S C C A}$ (vermelho) e $F_{|D C C A|}(a z u l)$, (a) magnitude, (b) sinal e (c) original. Observe que $0 F_{D F A}$ coincide com o $F_{|D C C A|}$. 
A Tabela 2 mostra a autocorrelação para a resistividade, onde a série de magnitude apresentou um comportamento persistente, enquanto que as séries originais e de sinais se apresentaram antipersistentes.

\begin{tabular}{|c|c|c|c|}
\hline$\rho(\Omega m)$ & $D F A$ & $S C C A$ & $D C C A$ \\
\hline Magnitude & 0,757 & 0,929 & 0,757 \\
\hline Sinal & 0,416 & 0,426 & 0,416 \\
\hline Original & 0,137 & 0,083 & 0,137 \\
\hline
\end{tabular}

Tabela 2: Expoentes de Hurst, para a autocorrelação para o perfil de resistividade do poço 1BAS121.

No caso da correlação cruzada entre os parâmetros físicos para os quatro poços, observou-se que as séries de magnitude foram sempre persistentes, enquanto que as séries de sinais e originais se apresentaram anti persistentes. A análise de correlação cruzada foi feita para os parâmetros físicos no mesmo poço e para os parâmetros físicos em diferentes poços. Este tipo de comportamento se apresentou para todos os quatro poços nos três perfis listados.

\section{Análise DCCA Local}

Para a análise local usando o coeficiente DCCA, foi utilizado 0 procedimento de janela deslizante (subconjunto de séries), de um determinado tamanho, ao longo das séries, e, calculamos $\sigma_{D C C A}(\mathrm{v})$ para cada janela. Se relacionarmos a janela $\vee$ com a profundidade $z$, podemos obter os coeficientes DCCA em função da escala $\vee$ e da profundidade $z, \sigma_{D C C A}(\vee, z)$. Foram avaliados os coeficientes de correlação cruzada DCCA para as séries de magnitude com uma janela em movimento com tamanho de 200 pontos.

Nas Figuras 6 e 7, a barra horizontal de cores dos diagramas, vai desde a cor preta até a vermelha, e está associada ao nível de correlação existente entre os sinais dados pelos valores de $\sigma_{D C C A}(\mathrm{v}, \mathrm{t})$, com um intervalo $-1 \leq$ $\sigma_{D C C A}(\mathrm{v}, \mathrm{t}) \leq 1$. Os sinais serão anticorrelacionados para valores negativos, passando por zero onde não há correlação e, por fim, para valores positivos onde os sinais são ditos correlacionados.

Tais resultados estão reacionados com os anteriormente obtidos nos expoentes DCCA de correlação cruzada, (Marinho et al., 2013). Note que $\lambda>0,5$, que representa um comportamento persistente, corresponde ao diagrama dos coeficientes do DCCA com eventos altamente correlacionados em todas as escalas para vários intervalos espaciais.

A Figura 6 mostra as correlações cruzadas, para o poço 1BAS68, entre os parâmetros físicos $D T, \gamma$ e $\rho$.
Observe que para as três correlações impostas para o poço 1BAS68 obteve-se diversos pontos em profundidade onde cada par de parâmetros físicos estão correlacionados. Resultados semelhantes foram obtidos para os outros três poços.

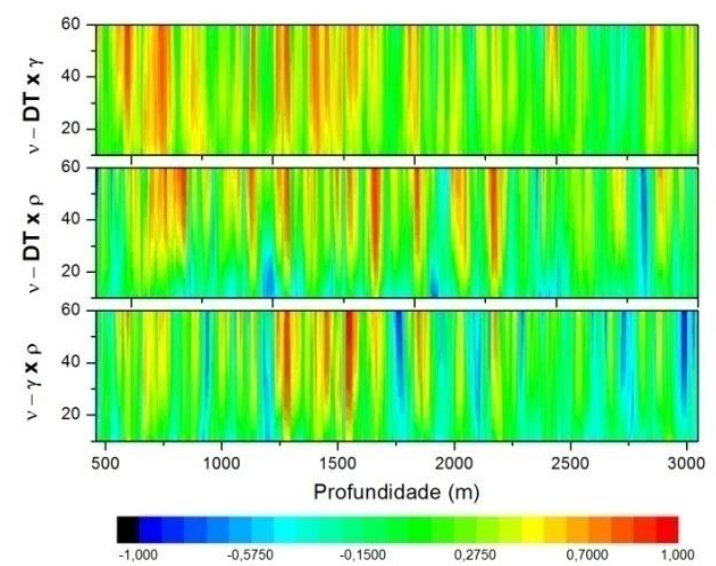

Figura 6: Diagrama dos coeficientes de correlação cruzada entre duas propriedades físicas distintas em função da profundidade, para o poço 1BAS68.

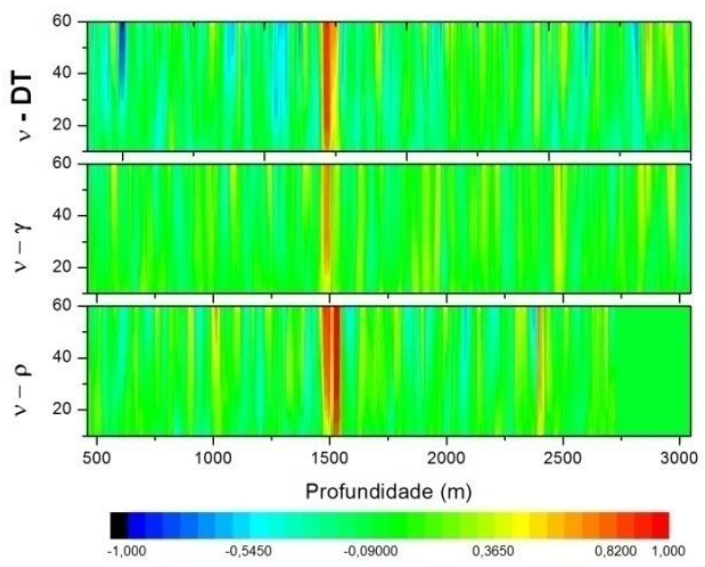

Figura 7: Diagrama dos coeficientes de correlação cruzada para a mesma propriedade física em função da profundidade, entre os poços 1BAS121 e 1BAS68.

A Figura 8 foi obtida por meio do procedimento da janela deslizante. Pode-se observar que nas coordenadas $(15 ; 1500)$ metros temos uma superposição de pontos para os três parâmetros físicos. Esta coincidência pode significar que a litologia do poço 1BAS121, na profundidade de 1500 metros, é semelhante à litologia do poço 1BAS68 que está deslocado 15 metros em relação ao poço 1BAS121, ou seja, estas camadas devem possuir as mesmas características físicas, e esta diferença vertical de 15 metros pode estar relacionada a um evento geológico ocorrido no passado. 


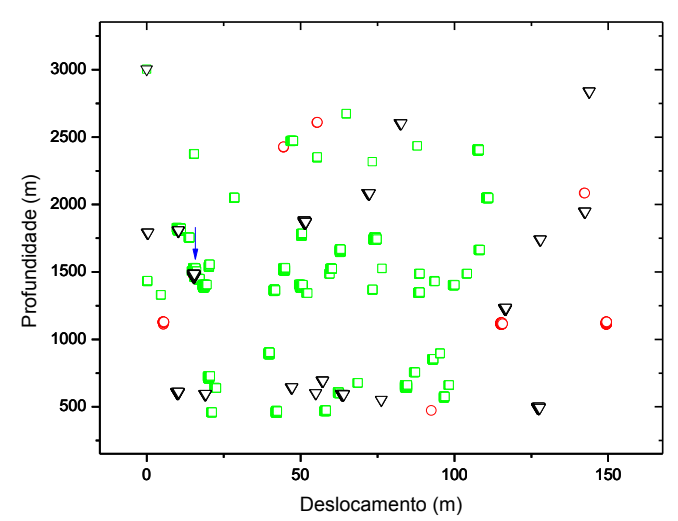

Figura 8: Diagrama com indicação de ocorrência de alta correlação cruzada $(\sigma \geq 0,7)$ na mesma propriedade física em função da profundidade e do deslocamento relativo, para os poços 1BAS68 e 1BAS121. A representação geométrica de cada parâmetro físico é dada por $D T=>\nabla, \gamma=>$ e $\rho=>0$.

O diagrama da Figura 7 mostra a correlação de um mesmo parâmetro físico entre os poços 1BAS121 e 1BAS68. Note que foi possível obter um ponto em comum, com maior intensidade na profundidade de 1500 metros, para um mesmo parâmetro físico. Este resultado pode ser comparado com o resultado do diagrama da Figura 8, confirmando a equivalência do deslocamento de 15 metros entre as camadas dos dois poços. Resultados semelhantes às Figuras 7 e 8 foram obtidos para os parâmetros físicos de todos os quatro poços.

É possível verificar, em um perfil de poço, vários marcos estratigráficos, marcos esses que podem ser correlacionados poço a poço. Isso pode ser verificado através das intensidades das amplitudes dos picos em cada perfil em função da profundidade, como o que foi mostrado na Figura 3.

Na Figura 9, relacionada ao poço 1BAS80, podemos visualizar diversos picos em toda extensão da profundidade para os perfis gama e resistividade. Estes picos, visualizados em pequenas e altas intensidades, ocorreram para os quatro poços estudados, se repetindo em diferentes intervalos espaciais para cada poço analisado, podendo indicar variações nas composições físicas e/ou químicas em camadas sedimentares, fazendo com que fosse possível correlacionar os quatro poços. Com isso, é possível identificar e interpretar se houve ou não algum acúmulo de sedimentos em uma dada região.

A mesma figura mostra que existe uma quebra no contraste, com aumento de intensidade, na radiação gama e resistividade a partir da profundidade de $2000 \mathrm{~m}$. Isto pode significar que a camada estratigráfica que se encontra a partir desta profundidade possui um maior teor de radiação gama e resistividade quando comparada com as camadas estratigráficas superiores. Uma possibilidade para a interpretação deste contraste seria um sistema de turbiditos, caraccterizados por camadas com grande continuidade lateral e acamamento regular, formados a partir de correntes de turbidez.

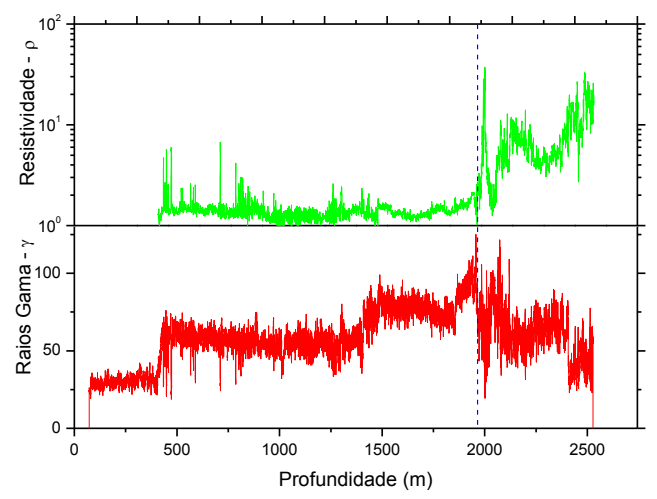

Figura 9: Identificação de contraste, indicada pela linha tracejada em azul, nos perfis gama e resistividade, para o poço 1BAS80.

A Figura 10 faz uma comparação entre os perfis sônico, gama e de resistividade para os poços 1BAS121 e 1BAS80, geradas a partir dos resultados obtidos em Marinho et al. (2013), Carvalho e Antunes (1988) e Henriques (2012). As regiões limitadas pelas linhas tracejadas em azul representam alguns intervalos de picos que foram observados nos resultados obtidos nos trabalhos citados. Nessa comparação entre um mesmo perfil para dois poços distintos, quando existe uma alta semelhança entre os sinais nos dois poços, em algum intervalo em profundidade, pode ser devido a uma mesma camada geológica.

A Figura 9 mostra uma estreita região em torno dos pontos em profundidade de $1500 \mathrm{~m}$ e $2450 \mathrm{~m}$ em comum para os dois poços, 1BAS121 e 1BAS80, para os três perfis analisados, conforme observado em Marinho et al. (2013). Com base em Carvalho e Antunes (1988), foi possível inferir através do poço 1BAS80 uma camada de calcilutitos em torno de $1450 \mathrm{~m}$, o mesmo ocorrendo no poço 1BAS121 na mesma profundidade. A mesma análise vale para a profundidade em torno do ponto de $2400 \mathrm{~m}$.

\section{Conclusões}

Os resultados mostraram que existem propriedades de escala para os três parâmetros físicos em cada um dos quatro poços estudados, conforme os expoentes de Hurst. As séries originais e de sinais se apresentaram antipersistentes para os métodos DFA e SCCA, enquanto que as séries de magnitude tiveram um comportamento persistente. Isso foi notado para os resultados baseados na autocorrelação e nas correlações cruzadas. 

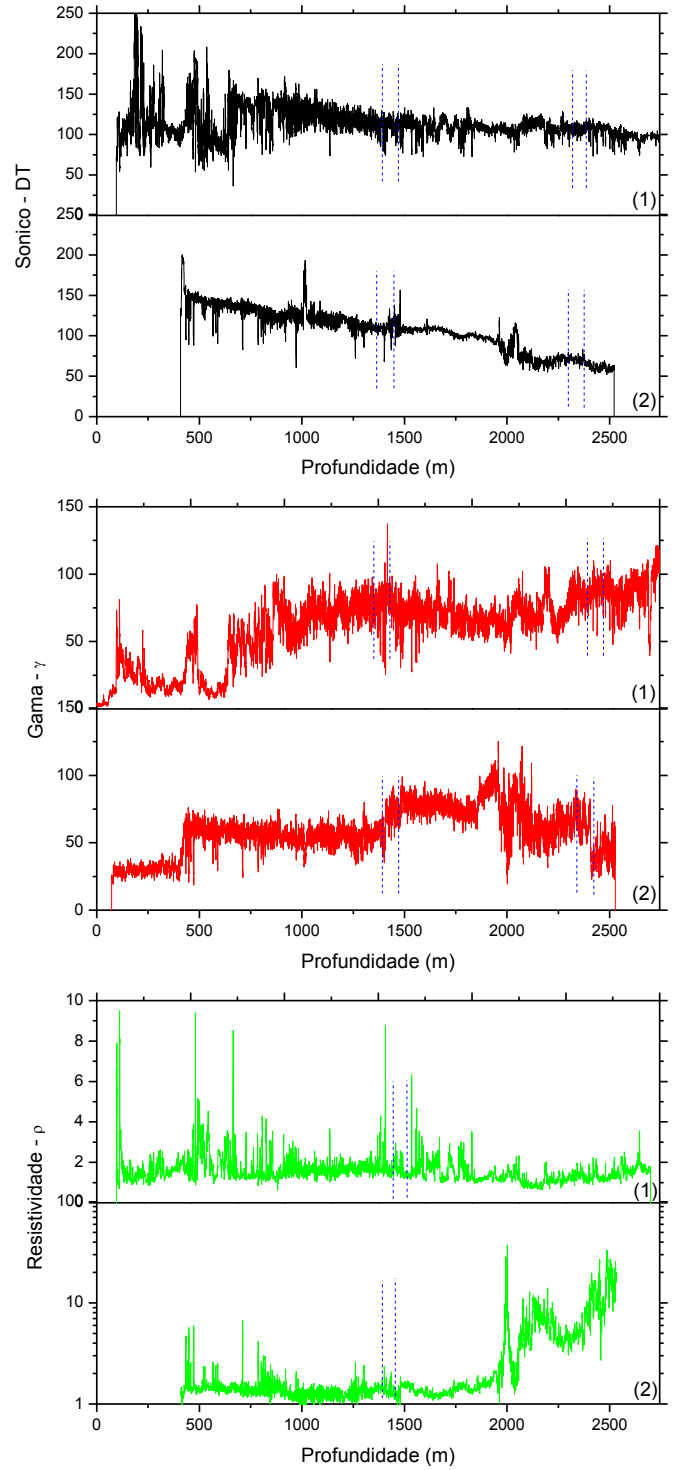

Figura 10: Comparação de uma mesma propriedade física entre os poços 1BAS121, legenda (1) e 1BAS80, legenda (2).

Com relação aos coeficientes, foi possível validar a técnica DCCA através da correlação entre camadas estratigráficas de cada par de poços estudados. Utilizando as correlações cruzadas através dos coeficientes DCCA, foram identificados deslocamentos verticais de uma mesma camada que passa por dois poços distintos, evidenciando um possível evento geológico. Por meio dos diagramas de poços e de trabalhos anteriores na literatura pode-se inferir a existência de litologias caracterizadas por camadas de calcilutitos.

\section{Agradecimentos}

Euler Marinho agradece a CAPES pela bolsa de doutorado. Amin Bassrei agradece ao $\mathrm{CNPq}$ e à
Universidade de Stanford pelo apoio à sua estada de um ano como professor visitante, e ao CNPq e à PETROBRAS pelo apoio ao projeto INCT-GP. Roberto Andrade agradece ao $\mathrm{CNPq}$ pelo apoio à pesquisa. Todos os autores agradecem à FINEP pelo apoio ao projeto Rede 01 . Os perfis de poços foram provenientes da PETROBRAS como contrapartida ao projeto FINEP Rede 01.

\section{Referências}

Carvalho, M. D. e Antunes, R. L., 1988, Calcilutitos do oligoceno da Bacia do Jequitinhonha com similaridade com os calcilutitos do marco azul da Bacia de Campos, Boletim de Geociências da PETROBRAS, Rio de Janeiro, 2: 291-292.

Dimri, V. P., 2005, Fractal Behavior of the Earth System, Springer, New York.

Feder, J., 1988, Fractals, Plenum Press, New York.

Goltz, C., 1998, Fractal and Chaotic Properties of Earthquakes, Springer, New York.

Hurst, H. E., 1951, Long-term storage capacity of reservoirs, Transactions of the American Society of Civil Engineers, 116: 770-808.

Henriques, M. V. C., 2012, Representações Espectrais de Sistemas Complexos: Aplicações à Síntese de Superfícies Brownianas Fracionárias Anisotrópicas, Filtragem de Sinais e Identificações de Correlações, Tese de Doutorado, Universidade Federal do Rio Grande do Norte.

Lowrie, W., 1997, Fundamentals of Geophysics, Cambridge University Press, New York.

Marinho, E. B. S.; Souza, A. M. Y. R; Andrade, R. F. S., 2013, Using detrended cross-correlation analysis in geophysical data, Physica A, 392: 2195-2201.

Podobnik, B.; Jiang, Z. Q.; Zhou, W. X. e Stanley, H. E., 2011, Statistical tests for power law cross-correlated processes, Physical Review E, 84: 066-118.

Peng, C. K.; Ashkenazy, Y.; Ivanov, P. C.; Havlin, S.; Goldberger, A. L.; Stanley, H. E., 2001, Magnitude and sign correlations in heartbeat fluctuations, Physical Review Letters, 86: 1900-1903.

Zebende, G. F.; Vassoler, R., 2012, DCCA crosscorrelation coefficient apply in time series of air temperature and air relative humidity, Physica A, 391: 2438-2443.

Zebende, G. F., 2011, DCCA cross-correlation coefficient: Quantifying level of cross-correlation, Physica A, 390: 614-618. 